\title{
Sulphonated polyaniline/MWCNTs nanocomposite: preparation and promising thermoelectric performance
}

\author{
Mahfoozurrahman Khan ${ }^{1} \cdot$ Tarique Anwer $^{1} \cdot$ Faiz Mohammad $^{1}$
}

Received: 1 April 2018 / Accepted: 31 July 2018 / Published online: 10 August 2018

(c) The Author(s) 2018

\begin{abstract}
Here is concise description of in situ prepared polyaniline and its nanocomposite with multi-walled carbon nanotubes followed by sulfonation. Thus, prepared materials were characterized by Fourier transform infrared spectroscopy, X-ray diffraction analysis, field emission scanning electron microscopy and electro-thermal analysis. Incorporation of ultrasonicated multi-walled carbon nanotubes significantly increased the electrical conductivity due to $\pi-\pi$ interaction of polyaniline with multi-walled carbon nanotubes and its back to back sulfonation further rendered fortification. Finally, as-prepared nanocomposite showed greater electrical conductivity as well as improved thermal stability in terms of DC electrical conductivity retention under isothermal and cyclic aging conditions.
\end{abstract}

Keywords Pani/MWCNTs nanocomposite $\cdot$ In situ polymerization $\cdot$ Sulfonation $\cdot$ Isothermal and cyclic aging

\section{Introduction}

The publication flood over 150 years old polyaniline and about 35 years aged rapidly rising carbon nanotubes [1, 2] evidently shows, how they have matured over the past years with a very broad spectrum and wide ranging from engineering and technological fields to their commercial and economical aspects till date. However, in spite of their astonishingly swift progress, both the polyaniline (Pani) and multi-walled carbon nanotubes (MWCNTs) have some limitations regarding their solubility and processibility [3]. To combat these problems, synergistic nanocomposite formulations of these constituents have been the burning desire of the researcher's world due to their outstanding mechanical and electronic properties [4]. Despite intractable improvement ensued so far, backbone stiffness of polyaniline, limited stability of MWCNTs dispersions in water remains an

Mahfoozurrahman Khan

mahfooz55@gmail.com

Tarique Anwer

tariqalig001@gmail.com

Faiz Mohammad

faizmohammad54@ rediffmail.com

1 Department of Applied Chemistry, Faculty of Engineering and Technology, Aligarh Muslim University,

Aligarh 202002, India ongoing challenge complicating its processing, management and ultimately the scope of their applications of these materials [5]. On account of these and to optimize their efficiency for use in various applications such as high strength nanofibres, sensors, nanoelectronic wires, it has become of immense interest to attach some functional parts on the constituents [6-8]. These modifications (covalent or noncovalent) either by organic or inorganic species in general and especially by sulfonic groups considerably enhance their stability and solubility while strong surface acidity making it highly useful for sophisticated electronic applications and excellent catalyst support for highly dispersed metal nanoparticles [9-12].

For this, various approaches followed to date are hydrothermal method [13], microwave-enhanced chemical modification [14], indirect chemical modifications [15], etc. which set in functional moieties like $-\mathrm{SO}_{3} \mathrm{H},-\mathrm{COOH}$, etc. on MWCNTs and generally at elevated temperature. But, herein we have tried to attach functional group on polyaniline as well as over its nanocomposites with MWCNTs by concentrated $\mathrm{H}_{2} \mathrm{SO}_{4}$ at ice temperature $0-5{ }^{\circ} \mathrm{C}$. Detailed studies of DC electrical conductivity retention were also done under isothermal and cyclic aging conditions to ascertain their stability for potential applications in latest sophisticated technologies. 


\section{Experimental section}

\section{Materials used}

Monomer "aniline" from E-Merck India Ltd. was purified by distilling twice before use. Multi-walled carbon nanotubes (MWCNTs) used in this study was purchased from Iljin Nano Tech, Seoul, Korea (diameter and average length were about $10-20 \mathrm{~nm}$ and $20 \mu \mathrm{m}$, respectively). Potassium persulphate (PPS), $\mathrm{HCl}$ (AR grade) and methanol were purchased from CDH India Ltd. and were used as received. Double distilled water was used in all the experimental procedures and washing.

\section{Preparation of Pani(EB) and Pani(EB)/MWCNTs nanocomposite}

The nanocomposite of Pani(EB)/MWCNTS was prepared by in situ oxidative polymerization of aniline. Aniline $(5 \mathrm{~mL})$ was dropwise added to $200 \mathrm{~mL}$ of $1 \mathrm{M} \mathrm{HCl}$ under continuous stirring. The suspension of ultrasonicated nanoparticles of MWCNTs $(0.15 \mathrm{~g})$ in $100 \mathrm{~mL}$ of $1 \mathrm{M} \mathrm{HCl}$ was added into aniline solution. The solution which act as an oxidant was obtained by dissolving $14.8 \mathrm{~g}$ of $\mathrm{K}_{2} \mathrm{~S}_{2} \mathrm{O}_{8}$ in $200 \mathrm{~mL}$ of $1 \mathrm{M} \mathrm{HCl}$. The polymerization of aniline was affected by dropwise adding the oxidant solution into the reaction mixture and stirring continuously for about $22 \mathrm{~h}$. The resultant greenish black slurry was filtered and washed thoroughly with $2.5 \mathrm{~L}$ double distilled water to remove excess acid as well as oxidant until filtrate became colorless. Thus, prepared nanocomposite was dedoped by aqueous ammonia (1 M) solution to convert it into emeraldine base (EB) form. Pani(EB)/MWCNTs were dried at $70 \pm 5{ }^{\circ} \mathrm{C}$ for $2 \mathrm{~h}$ in an air oven, converted into fine powder and was stored in an airtight sample tube for further investigations. Pani(EB) was also prepared using the same method.

\section{$\mathrm{H}_{2} \mathrm{SO}_{4}$ treatment of Pani(EB) and Pani(EB)/MWCNTs nanocomposite}

Thus, prepared Pani(EB) and Pani(EB)/MWCNTs nanocomposite were treated by sulfuric acid using the earlier reported method [16]. As-prepared Pani(EB) and Pani(EB)/MWCNTs nanocomposite were treated with $\mathrm{H}_{2} \mathrm{SO}_{4}(1 \mathrm{M})$ in an ice bath $0-5{ }^{\circ} \mathrm{C}$ under constant stirring for $4 \mathrm{~h}$. The resultant greenish suspension was filtered, washed with water followed by $500 \mathrm{~mL}$ of methanol and dried in an air oven at $70 \pm 5{ }^{\circ} \mathrm{C}$ The as-prepared Pani(EB) and Pani(EB)/MWCNTs nanocomposite were assigned the names as S-Pani and S-Pani/MWCNTs, respectively.

\section{Characterization}

Surface morphologies of gold coated Pani(EB), Pani(EB)/ MWCNTs, S-Pani and S-Pani/MWCNTs were viewed under scanning electron microscope (LEO 435-VF). Their phase composition was analyzed for XRD patterns recorded by Bruker $\mathrm{D} 8$ diffractometer with $\mathrm{Cu} \mathrm{K} \alpha$ radiation at $1.540 \AA$ in the range of $5^{\circ} \leq 2 \theta \leq 70^{\circ}$ at $40 \mathrm{kV}$. The FTIR spectra were recorded using Perkin-ElmerSpectrum 2000 Spectrophotometer in $\mathrm{KBr}$ between 400 and $4000 \mathrm{~cm}^{-1}$. Electrical conductivity as well as thermal stability in terms of DC electrical conductivity retention was studied according to the method already reported [17] using the equation:

$\sigma=[\ln 2(2 S / W)] /[2 \pi S(V / I)]$,

where $I, V, W$ and $S$ are the current (A), voltage (V), thickness of the pellet $(\mathrm{cm})$ and probe spacing $(\mathrm{cm})$, respectively, and $\sigma$ is the DC electrical conductivity $\left(\mathrm{S} \mathrm{cm}^{-1}\right)$ [17]

\section{Results and discussion}

\section{Preparation of S-Pani and S-Pani/MWCNTS nanocomposite}

The proposed balanced chemical equation and formation scheme of Pani and Pani/MWCNTs nanocomposite and their treatment with $\mathrm{H}_{2} \mathrm{SO}_{4}$ have been summarized in Fig. 1 . Pani(EB) was prepared by simple oxidative polymerization and Pani(EB)/MWCNTs nanocomposite was also prepared same way by the additional use of MWCNTs. Some portion of these in situ products was converted to emeraldine salt (ES) form and a portion of it was treated with $1 \mathrm{M} \mathrm{H}_{2} \mathrm{SO}_{4}$ at ice temperature. On treating the EB form of Pani and Pani/ MWCNTs with sulfuric acid, a dark green free flowing powder was obtained after filtration, washing and drying. The chemical reaction schemes after treatment of $\mathrm{H}_{2} \mathrm{SO}_{4}$ it form $\mathrm{S}$-Pani and S-Pani(EB)/MWCNTs are given in the Figs. 1 and 2 , respectively.

The sulphuric acid-treated Pani(EB) and Pani(EB)/MWCNTs were exposed to $1 \mathrm{M}$ ammonia solution. It was observed that the color of the materials remained greenish, i.e., the materials did not undergo dedoping and did not form their corresponding bases. No change in color from greenish to blue was observed on heating the ammonia treated materials around $100{ }^{\circ} \mathrm{C}$. These observations suggest that the sulfonic groups have been attached to the benzene rings of the polyaniline. The materials were strongly doped to form very 
Fig. 1 Formation scheme of $\mathrm{H}_{2} \mathrm{SO}_{4}$-treated polyaniline (S-Pani)
Step-1: Preparation of Polyaniline (Emeraldine Salt or ES)

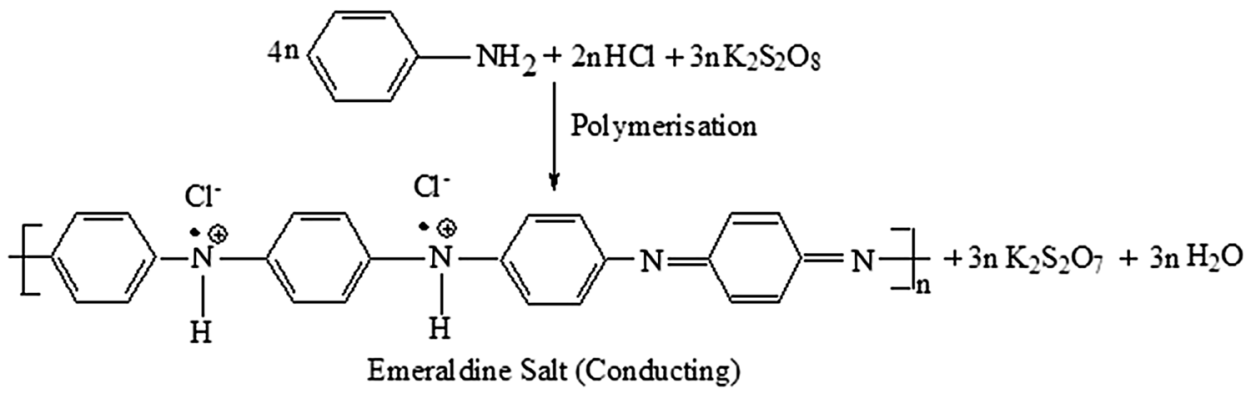

Step-2: Preparation of Polyaniline (Emeraldine Base or EB)
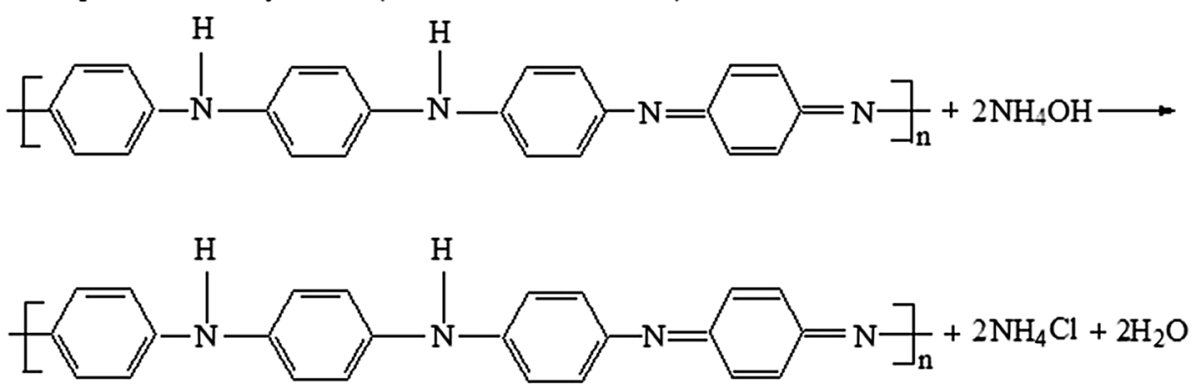

Emeraldine Base (Non conducting)

Step-3: $\mathrm{H}_{2} \mathrm{SO}_{4}$ Treatment of Polyaniline (Emeral dine Base or EB)

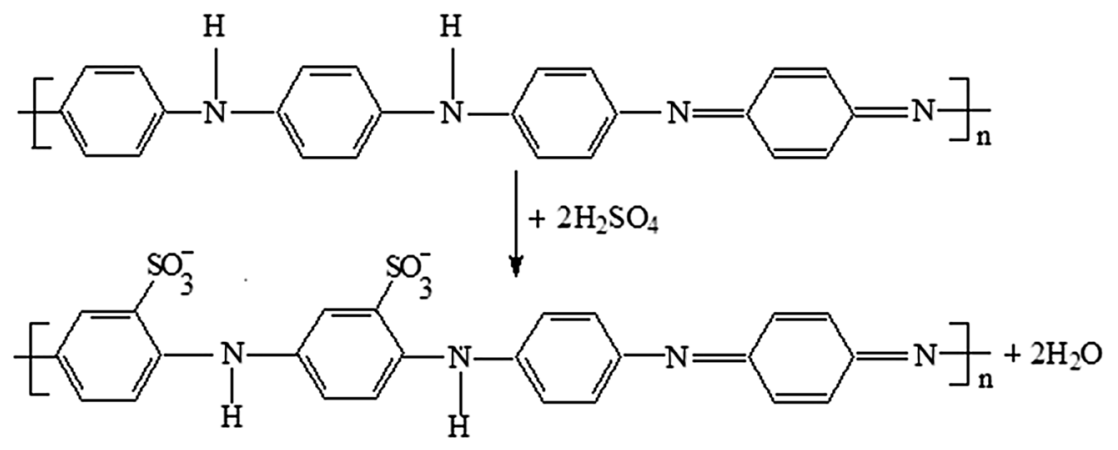

(S-Pani) stable sulphanilic type zwitterions structure (Fig. 3) which does not interact with ammonia and thus do not undergo dedoping. They have structures analogous to amino acids [18].

\section{FTIR spectroscopic studies}

FTIR spectra of Pani(EB) and Pani(EB)/MWCNTs nanocomposite and their $\mathrm{H}_{2} \mathrm{SO}_{4}$-treated products are shown in Fig. 4. For Pani, the main peaks at 1590 and $1485 \mathrm{~cm}^{-1}$ may be assigned to the $\mathrm{C}=\mathrm{C}$ stretching mode of vibrations of quinoid and benzenoid rings, respectively, which indicate the formation of emeraldine base form of polyaniline. Besides, peaks at higher wave numbers at 3230 and $2925 \mathrm{~cm}^{-1}$ may be attributed to the free (non-hydrogen bonded) $\mathrm{N}-\mathrm{H}$ stretching vibration and asymmetric vibrational stretching of $-\mathrm{CH}$, respectively [19]. Conversely, the peak at lower wave number at $1302 \mathrm{~cm}^{-1}$ corresponds to $\mathrm{C}-\mathrm{N}$ stretching vibration in the aromatic region and peak at $1132 \mathrm{~cm}^{-1}$ refers to $\mathrm{C}=\mathrm{N}$ stretching $(-\mathrm{N}=$ quinoid $=\mathrm{N}-)$, respectively. The last and lowest wave number peak noticed at $829 \mathrm{~cm}^{-1}$ is usually attributed to $\mathrm{C}-\mathrm{H}$ out-of plane bending vibration of 1,4-disubstituted benzenoid rings confirming the formation of Pani. For Pani(EB)/MWCNTs nanocomposite, almost all the FTIR peaks are similar to those of Pani and no significant difference was observed. This suggests that the inclusion of MWCNTs does not cause any significant alteration in polymer backbone structure. However, in comparison to Pani, one of the peaks of the nanocomposite exhibited a blue shift, i.e., the band at $1590 \mathrm{~cm}^{-1}$ slightly shifted to $1592 \mathrm{~cm}^{-1}$ revealing weak $\pi-\pi$ interaction of Pani with MWCNTs which is also supported by the findings of Zengin et al. [20]. 
Step-1: Preparation of $\mathrm{Pani}(\mathrm{ES}) / \mathrm{MWCN}$ Ts

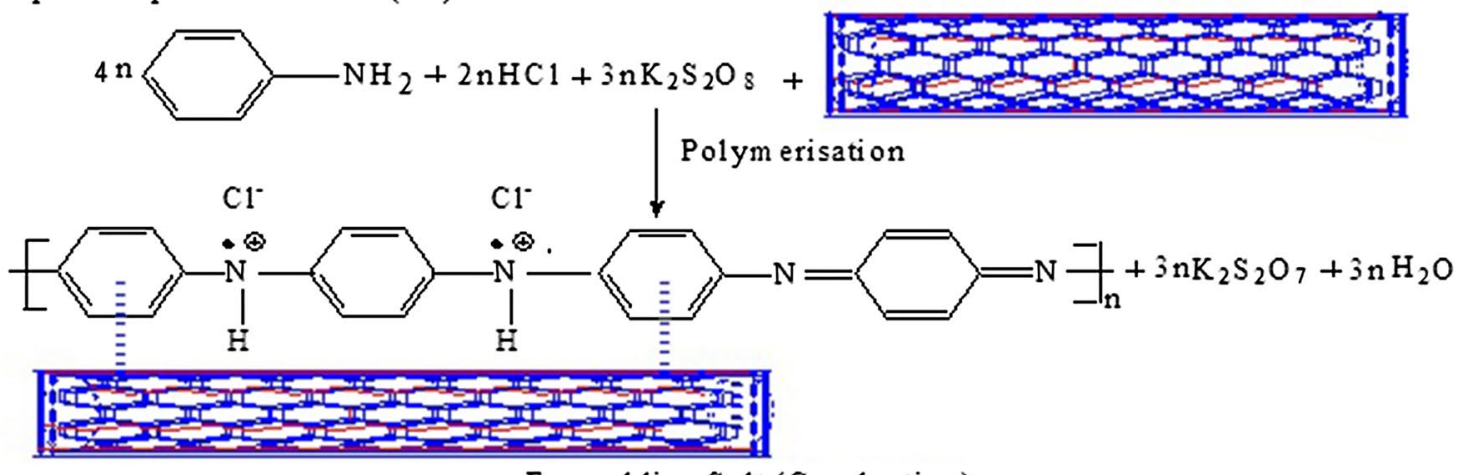

Em eraldine Salt (Conducting)

Step-2: Preparation of $\mathrm{Pani}(\mathrm{E} B) / \mathrm{MWCNTs}$
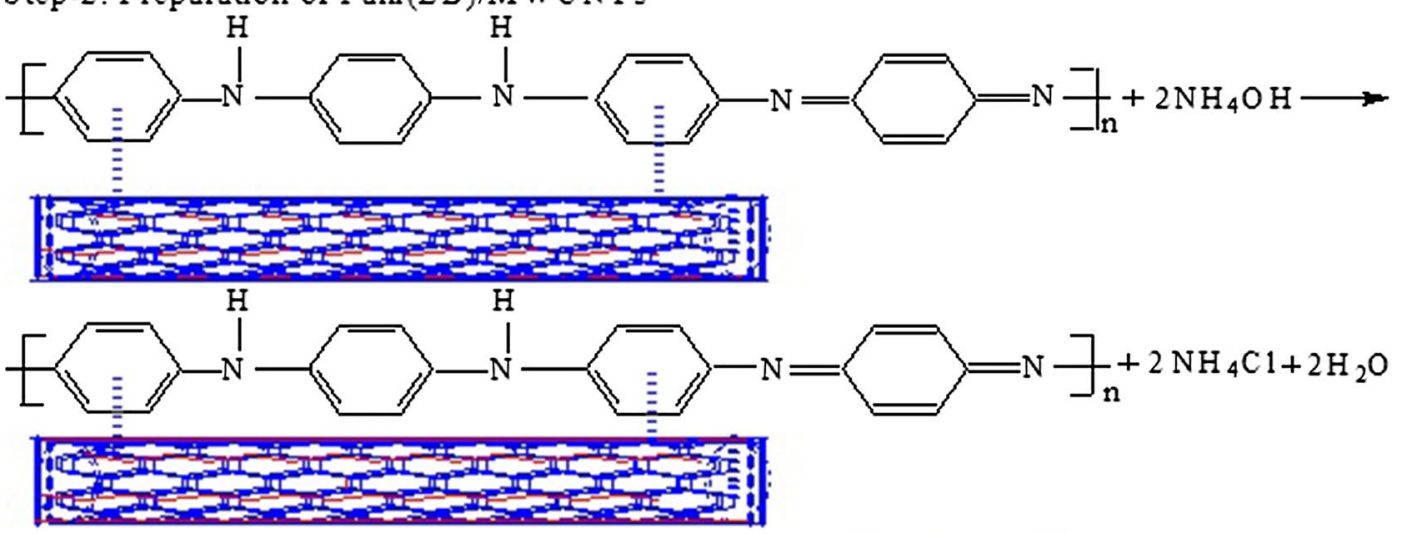

Em eraldine $B$ ase ( $N$ on-Conducting)

Step-3: $\mathrm{H}_{2} \mathrm{SO}_{4}$ Treatm ent of $\mathrm{Pani}(\mathrm{EB}) / \mathrm{MWCNT}$ s
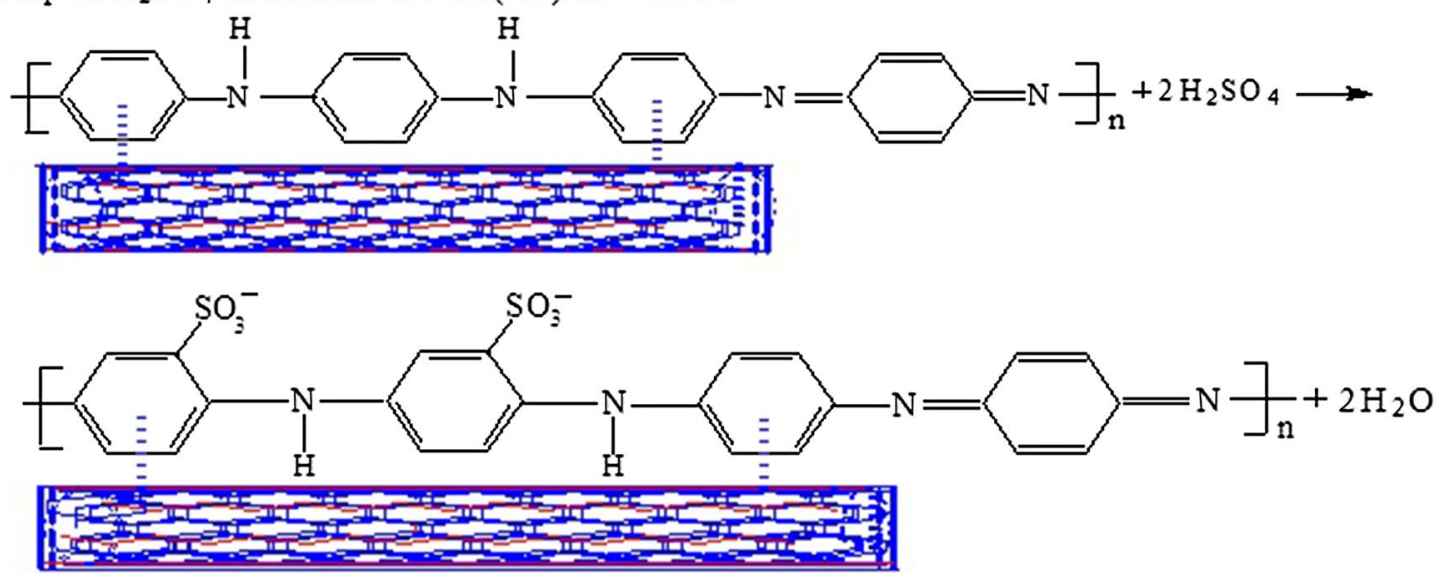

S-Pani/M W CNTs

Fig. 2 Formation scheme of $\mathrm{H}_{2} \mathrm{SO}_{4}$-treated Pani/MWCNTs (S-Pani/MWCNTs)

In case of $\mathrm{H}_{2} \mathrm{SO}_{4}$-treated form of Pani (S-Pani) as well as their nanocomposite (S-Pani/MWCNTs), almost all the vibrational peaks are much diffused. The characteristic peaks at 1578 and $1090 \mathrm{~cm}^{-1}$ may be attributed to $\mathrm{SO}_{3}$ asymmetric and symmetric stretching mode of vibration, respectively. Furthermore, peaks at 952 and $805 \mathrm{~cm}^{-1}$ correspond to the out-of-plane bending in a meta-substituted aromatic ring.

\section{X-ray diffraction studies}

X-ray diffractograms of Pani(EB), Pani(EB)/MWCNTs nanocomposite and their $\mathrm{H}_{2} \mathrm{SO}_{4}$-treated products are shown in Fig. 5 for phase analysis. In Fig. 5a, characteristic peaks appearing at $2 \theta=15.2,20.11$ and 26.5 , respectively, correspond to (011) (020) and (200) crystal planes of polyaniline 
Fig. 3 Stable sulphanilic type zwitterions structure of: a S-Pani and b S-Pani/MWCNTs

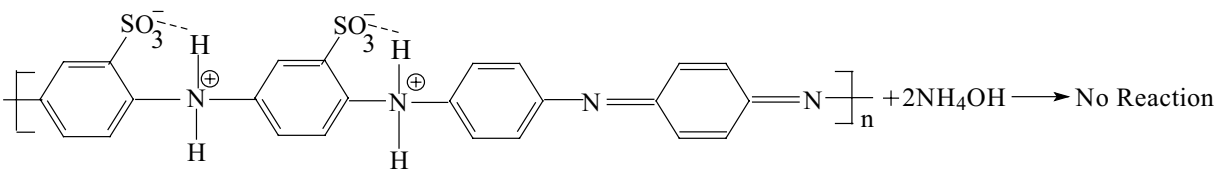

(a) Zwitterionic structure of S-Pani

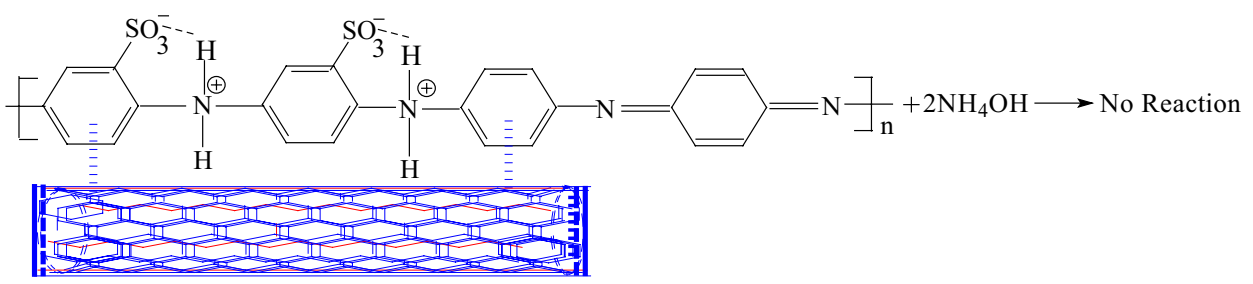

(b) Zwitterionic structure of S-Pani/MWCNTs

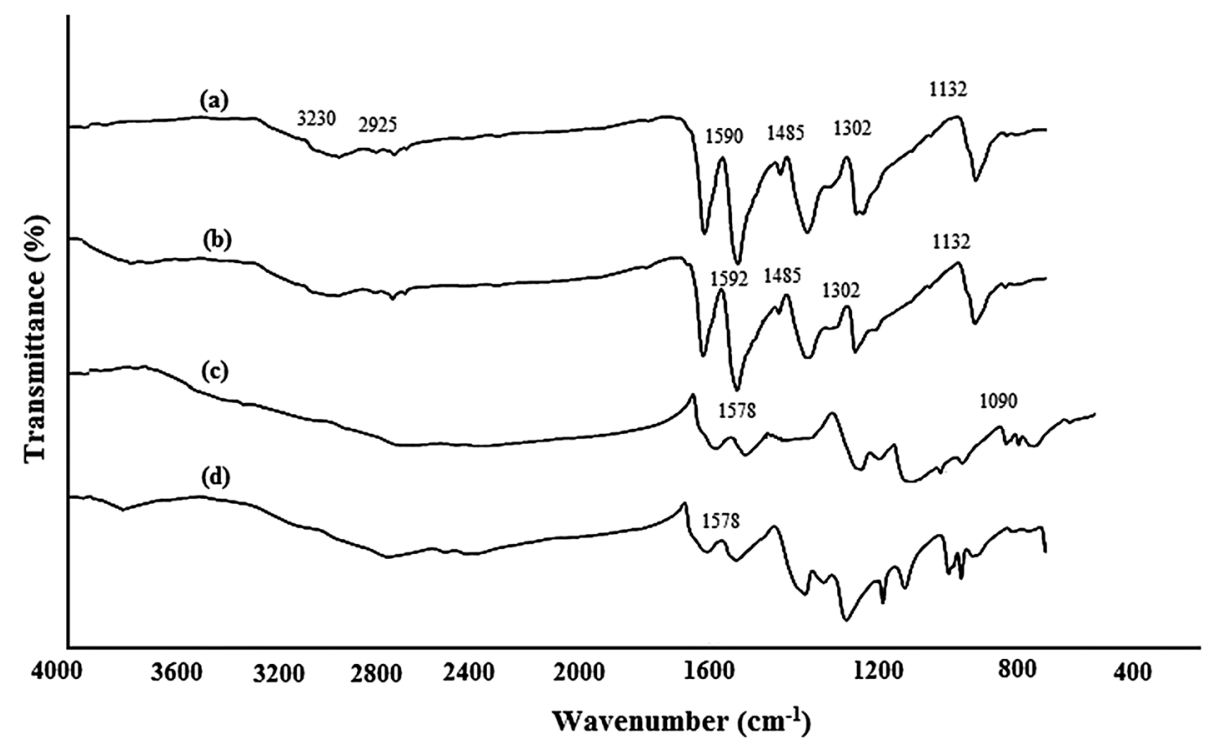

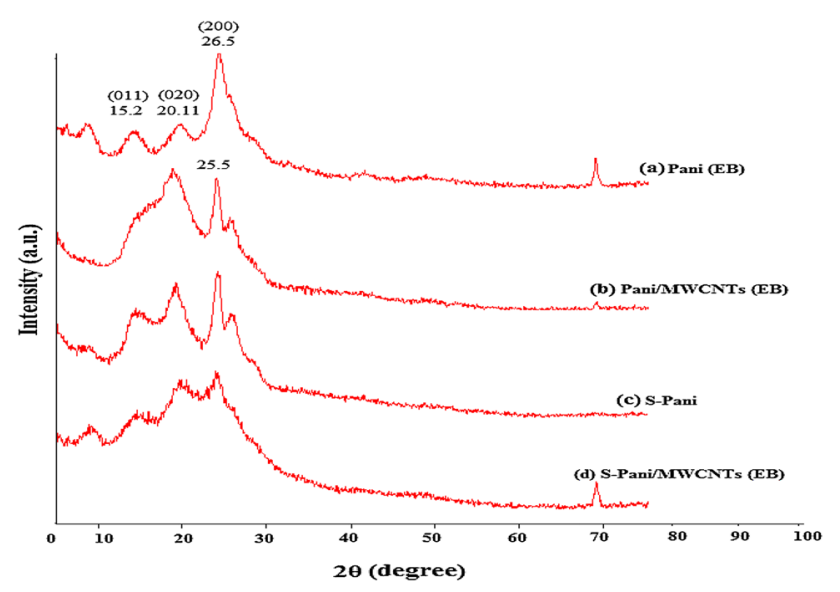

Fig. 5 XRD spectra of: a Pani (EB), b Pani/MWCNTs (EB), c S-Pani and d S-Pani/MWCNTs

[21]. For Pani(EB)/MWCNTS nanocomposite (Fig. 5b), XRD peaks are slightly diffused and broad with an additional sharp peak bearing shoulder at $2 \theta=25.5^{\circ}$. This seems to be an indicative of the presence of MWCNTs in the nanocomposite and suggests that the inclusion of MWCNTs enhances the amorphosity of the polymer backbone via $\pi-\pi$ interaction of Pani with MWCNTs.

In case of $\mathrm{H}_{2} \mathrm{SO}_{4}$-treated form of Pani (S-Pani) (Fig. 5c), as well as their nanocomposite (S-Pani/MWCNTs) (Fig. 5d), almost all the diffraction peaks are much diffused with very low intensity, indicating enhancement in amorphosity. Thus, results of XRD seem to support the possibility of sulfonation of these in situ products which was also implied by FTIR results.

\section{Scanning electron microscopy (SEM) studies}

Surface morphologies of Pani(EB), Pani(EB)/MWCNTs nanocomposite and their $\mathrm{H}_{2} \mathrm{SO}_{4}$-treated products are shown in Fig. 6 at different magnifications. Here, Pani (EB) shown in Fig. 6a seems to have three-dimensional particulate and 
Fig. 6 SEM micrographs of: a Pani (EB), b Pani(EB)/MWCNTs, c S-Pani and d S-Pani/ MWCNTs

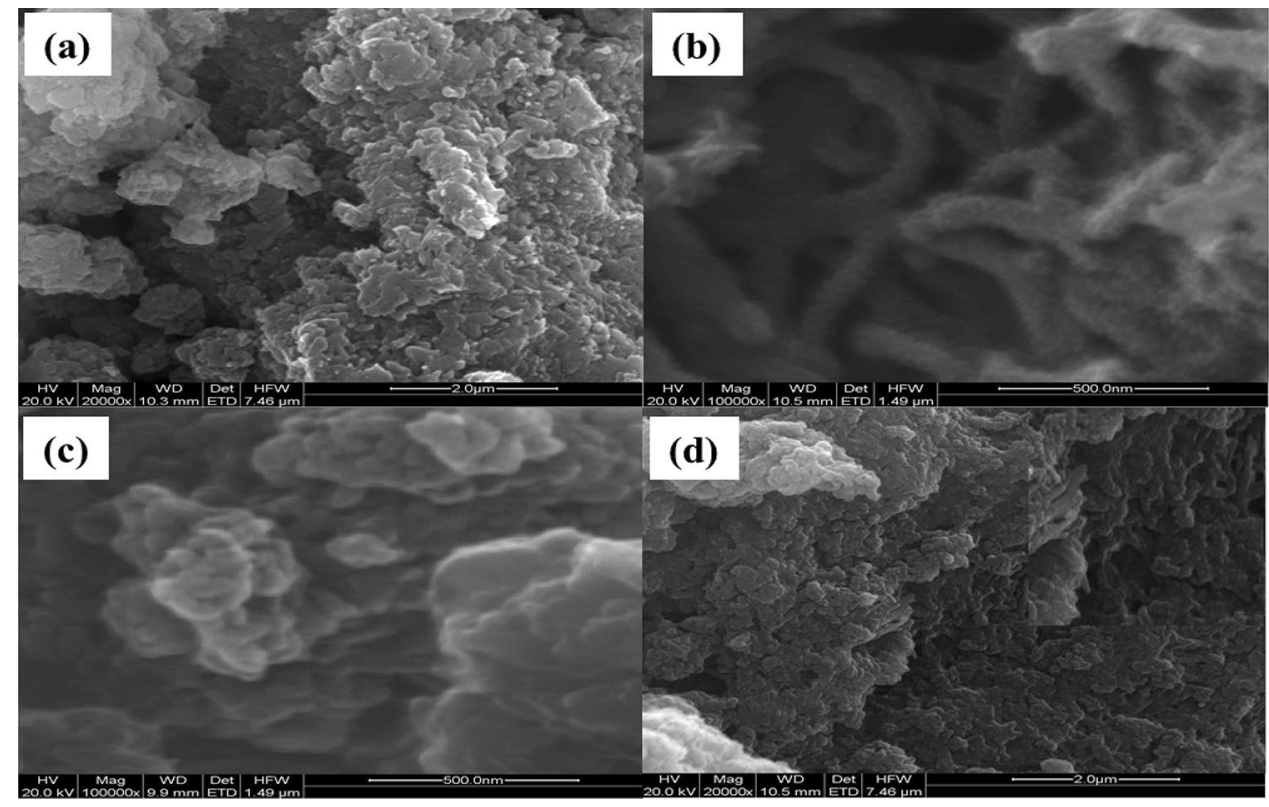

somewhat flaky structure which are common in ordinary polyanilines. Whereas in the case of Pani/MWCNTS nanocomposites (Fig. 6b), the particulate Pani is spread over the surface of MWCNTs. While in Fig. 6c, d globular structures are also being observed which seems to be because of the attachment of $-\mathrm{SO}_{3}{ }^{-}$group in these in situ products. Figure $6 \mathrm{~d}$ presents network of tubular structures randomly distributed with some globular heads which might act as a new conducting bridge to enhance the conductivity of $\mathrm{H}_{2} \mathrm{SO}_{4}$-treated nanocomposite.

\section{DC electrical conductivity}

The electro-thermal studies' as-prepared in situ products can be measured only ES form of Pani(ES) and Pani(ES)/ MWCNTs. While EB form of Pani as well as Pani/MWCNTs shows conductivity after sulfonation to form S-Pani and S-Pani/MWCNTs were very well done by standard 4-in-line probe technique. From the electrical conductivity measured, these materials were observed to behave as semiconductors within the temperature range of $40-150{ }^{\circ} \mathrm{C}$. It was observed that the addition of MWCNTs has caused enhancement in electrical conductivity in as-prepared Pani(ES)/MWCNTs nanocomposite. Since both the Pani and MWCNTs are good conductors, the enhancement in DC electrical conductivity may be credited to the additive synergism of both the constituents interacting at molecular level. Further treatment with $\mathrm{H}_{2} \mathrm{SO}_{4}$ also caused promising augmentation in electrical conductivity of S-Pani as well as S-Pani/MWCNTs. However, the higher electrical conductivity of S-Pani/ MWCNTs compared with S-Pani can basically be attributed to the extra presence of MWCNTs in the nanocomposite and its back to back internal doping with sulfonic groups further rendered enrichment.

Herein, it seems that $-\mathrm{SO}_{3}{ }^{-}$groups get covalently attached to Pani in S-Pani leading to internal doping and causing very high increment in electrical conductivity. Likewise, in case of S-Pani/MWCNTs, $-\mathrm{SO}_{3}{ }^{-}$groups get attached on Pani of S-Pani/MWCNTs via covalent bond and simultaneously dope it. The formation of intact covalent bond also increases significantly its thermal stability. Thus, $\mathrm{H}_{2} \mathrm{SO}_{4}$ treatment of as-prepared Pani has increased its conductivity $\sim$ almost 18 times and of Pani/MWCNTs about 20 times. Besides, the $\pi-\pi$ interaction between the $\pi$-bonded surface of the MWCNTs and the conjugated $s p^{2}$-hybridized rings of Pani also imparts the enhancement of the conductivity up to some extent (Fig. 7).

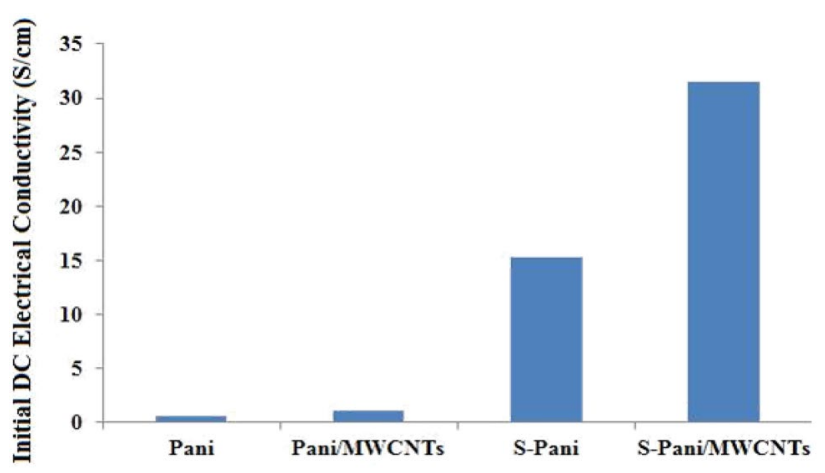

Fig. 7 Initial electrical conductivities of Pani(ES), Pani(ES)/MWCNTs, S-Pani and and S-Pani/MWCNTs 


\section{DC electrical conductivity retention}

For better understanding of effect of accelerated aging on the DC electrical conductivity retention of these as-prepared materials, they were examined by isothermal as well as cyclic accelerated aging techniques.

\section{Stability under isothermal aging conditions}

The change in relative electrical conductivity during each experiment was divided by the duration of the experiment (40 min) to get electrical conductivity loss/gain per minute of heating as given by the following equation:

$\Delta \sigma=\frac{\sigma_{\mathrm{f}}-\sigma_{\mathrm{i}}}{t}$,

where $\Delta \sigma$ is the change in relative electrical conductivity/ $\min , \sigma_{\mathrm{f}}$ is the final relative electrical conductivity at temperature $T, \sigma_{\mathrm{i}}$ is the initial relative electrical conductivity at temperature $T$ and $t$ is the duration of experiment (40 $\mathrm{min}$ ).

The stability in terms of DC electrical conductivity retention of Pani(ES), Pani(ES)/MWCNTs, S-Pani and S-Pani/ MWCNTs was studied under isothermal aging conditions as shown in Fig. 8. It may be observed that the electrical conductivity loss per minute increases with increase in the temperature of the isothermal aging experiment. The maximum loss in electrical conductivity per minute was observed in case of Pani(ES)/MWCNTs nanocomposite following the same trend. On the other hand, S-Pani/MWCNTs nanocomposite showed minimum loss in DC conductivity per minute under isothermal aging conditions. It may be inferred that the $\mathrm{H}_{2} \mathrm{SO}_{4}$ treatment of Pani(ES)/MWCNTs induced thermal stability in the nanocomposite besides enhancing its DC electrical conductivity to a great extent. In contrast to $\mathrm{HCl}$ dopant, it seems that sulfonic acid groups attached to the polymer by covalent bonds leading to internal doping of the polymer do not volatilize at elevated temperature Fig. 9.

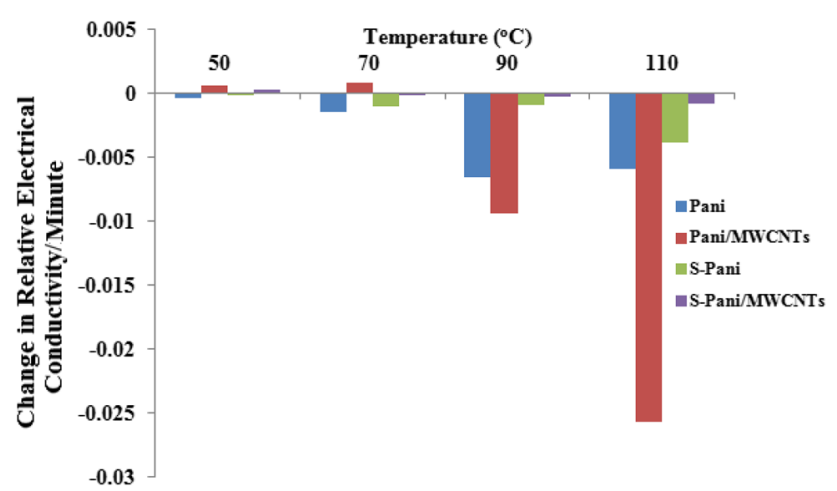

Fig. 8 Change in relative electrical conductivity/minute of Pani(ES), Pani(ES)/MWCNTs, S-Pani and S-Pani/MWCNTs nanocomposite under isothermal aging conditions

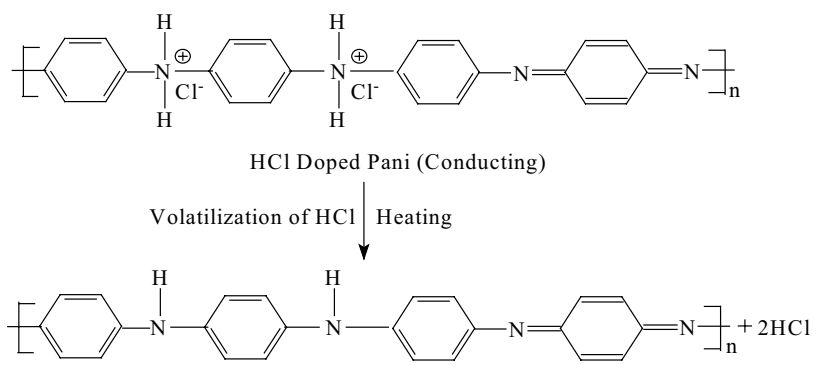

(a) Undoped Pani (Non Conducting)

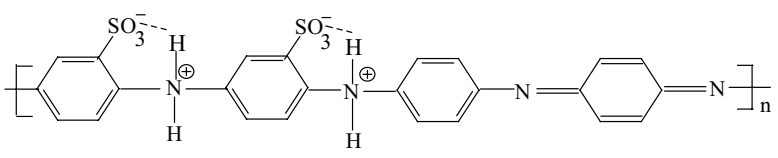

Zwitterionic Structure of S-Pani (Doped and Conducting)

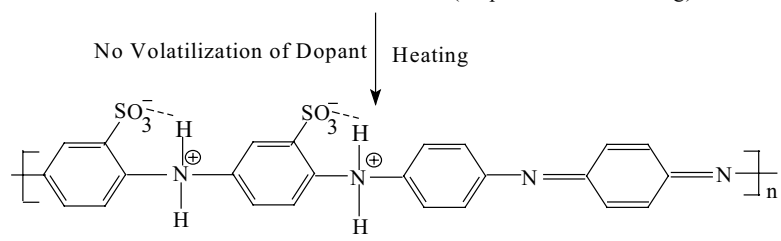

(b) Zwitterionic Structure of S-Pani (Doped and Conducting)

Fig. 9 Effect of heating on a Pani (ES) and b zwitterionic structure of S-Pani

Thus, the combined effect of incorporation of MWCNTs into Pani following sulfuric acid treatment enhances the stability in terms of retaining electrical conductivity leading to better stabilization of S-Pani/MWCNTs.

\section{Stability under cyclic aging conditions}

The stability in terms of DC electrical conductivity retention of Pani(ES), Pani(ES)/MWCNTs, S-Pani and S-Pani/ MWCNTs was also studied by cyclic aging technique within the temperature range from 40 to $150^{\circ} \mathrm{C}$. It may be observed

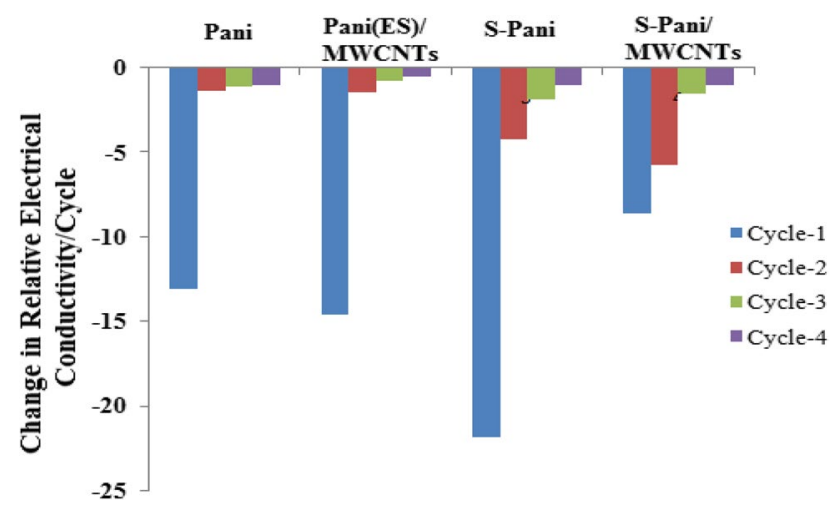

Fig. 10 DC electrical conductivity retention of Pani(ES), Pani(ES)/ MWCNTs, S-Pani and S-Pani/MWCNTs nanocomposite under cyclic aging conditions 
from Fig. 10 that both Pani(ES) and Pani(ES)/MWCNTs showed a huge loss in electrical conductivity in first cycle which seems to be because of loss of moisture and volatile components present in the sample. But after that there is a constant decrease in loss leading towards constancy.

Likewise, S-Pani as well as S-Pani/MWCNTs showed a regular decrease in loss in electrical conductivity in subsequent cycles causing great improvement in thermal stability. This seems to be because of the development of highly strong covalent bond between $-\mathrm{SO}_{3} \mathrm{H}$ groups of $\mathrm{H}_{2} \mathrm{SO}_{4}$ with Pani.

\section{Conclusion with future prospects}

In summary, we have successfully prepared Pani and its nanocomposite with MWCNTs via oxidative polymerization. Furthermore, the effective supplementation of sulfuric acid has brightly improved their electrical properties. After detailed studies of characterizations and electrical properties, finally as-prepared S-Pani/MWCNTs nanocomposite was observed to possess greater electrical conductivity and better isothermal stability in terms of electrical conductivity retention than Pani and S-Pani. This increment is attributed to the addition of ultrasonicated MWCNTs in the polymer matrix, and its back to back treatment with sulfuric acid further rendered enrichment. Thus, it may be postulated as a universal approach to prepare nanocomposite with enhanced electrical conductivity as well as enriched thermal stability which may find better realistic applications in modern electronic devices and seems to be a replaceable alternate even for metals in next generation.

Acknowledgements One of the authors, Mahfoozurrahman Khan, acknowledges with thanks the financial support from University Grants Commission (UGC) New Delhi.

Open Access This article is distributed under the terms of the Creative Commons Attribution 4.0 International License (http://creativeco mmons.org/licenses/by/4.0/), which permits unrestricted use, distribution, and reproduction in any medium, provided you give appropriate credit to the original author(s) and the source, provide a link to the Creative Commons license, and indicate if changes were made.

\section{References}

1. Dong, B., He, B.L., Xu, C.L., Li, H.L.: Preparation and electrochemical characterization of polyaniline/multi-walled carbon nanotubes composites for supercapacitor. Mater. Sci. Eng. B 143, 7-13 (2007)

2. Gospodinova, N., Terlemezyan, L.: Conducting polymers prepared by oxidative polymerization: polyaniline. Prog. Polym. Sci. 23, 1443-1484 (1998)

3. Subrahmanyam, A.R., Geetha, V., Kumar, A., Zhang, S., Kumar, J.S.: Mechanical and electrical conductivity studies of PANI-PVA and PANI-PEO blends. Int. J. Mater. Sci. 2, 27-30 (2012)
4. Ansari, M.O., Mohammad, F.: Thermal stability and electrical properties of dodecyl-benzene-sulfonic-acid doped nanocomposites of polyaniline and multi-walled carbon nanotubes. Compos. Part B Eng. 43, 3541-3548 (2012)

5. Lin, Y.W., Wu, T.M.: Synthesis and characterization of externally doped sulfonated polyaniline/multi-walled carbon nanotube composites. Compos. Sci. Technol. 69, 2559-2565 (2009)

6. Huang, J., Virji, S., Weiller, B.H., Kaner, R.B.: Polyaniline nanofibers: facile synthesis and chemical sensors. J. Am. Chem. Soc. 125, 314-315 (2003)

7. Song, E., Choi, J.W.: Conducting polyaniline nanowire and its applications in chemiresistive sensing. Nanomaterials 3, 498-523 (2013)

8. Huang, L., Wang, Z., Wang, H., Cheng, X., Mitra, A., Yan, Y.: Polyaniline nanowires by electropolymerization from liquid crystalline phases. J. Mater. Chem. 12, 388-391 (2002)

9. Bai, H., Xu, Y., Zhao, L., Li, C., Shi, G.: Non-covalent functionalization of graphene sheets by sulfonated polyaniline. Chem. Commun. 10, 1667-1669 (2009)

10. Koul, S., Dhawan, S.K., Chandra, R.: Compensated sulphonated polyaniline-correlation of processibility and crystalline structure. Synth. Met. 124, 295-299 (2001)

11. Drelinkiewicz, A., Zięba, A., Sobczak, J.W., Bonarowska, M., Karpinski, Z., Waksmundzka-Gora, A., Stejskal, J.: Polyaniline stabilized highly dispersed Pt nanoparticles: preparation characterization and catalytic properties. React. Funct. Polym. 69, 630-642 (2009)

12. Wei, X., Epstein, A.J.: Synthesis of highly sulfonated polyaniline. Synth. Met. 74, 123-125 (1995)

13. Cai, H., An, X., Cui, J., Li, J., Wen, S., Li, K., Shen, M., Zheng, L., Zhang, G., Shi, X.: Facile hydrothermal synthesis and surface functionalization of polyethyleneimine-coated iron oxide nanoparticles for biomedical applications. ACS Appl. Mater. Interfaces 5, 1722-1731 (2013)

14. Hatamzadeh, M., Mahyar, A., Jaymand, M.: Chemical modification of polyaniline by $\mathrm{N}$-grafting of polystyrenic chains synthesized via nitroxide-mediated polymerization. J. Braz. Chem. Soc. 23, 1008-1017 (2012)

15. Zhao, H.B., Yang, J., Lin, T.T., Lu, Q.F., Chen, G.: Nanocomposites of sulfonic polyaniline nanoarrays on graphene nanosheets with an improved supercapacitor performance. Chem. A Eur. J. 21, 682-690 (2015)

16. Yue, J., Epstein, A.J.: Synthesis of self-doped conducting polyaniline. J. Am. Chem. Soc. 112, 2800-2801 (1990)

17. Ansari, M.O., Mohammad, F.: Thermal stability, electrical conductivity and ammonia sensing studies on p-toluenesulfonic acid doped polyaniline: titanium dioxide (pTSA/Pani: $\mathrm{TiO}_{2}$ ) nanocomposites. Sens. Actuators B Chem. 157, 122-129 (2011)

18. Morrison, R.T., Boyd, R.N.: Organic Chemistry, 6th Edition, Prentice Hall Pvt. Ltd., New Delhi (1992)

19. Pahovnik, D., Zagar, E., Kogej, K., Vohlidal, J., Zigon, M.: Polyaniline nanostructures prepared in acidic aqueous solutions of ionic liquids acting as soft templates. Eur. Polym. J. 49, 13811390 (2013)

20. Kondawar, S.B., Anwane, S.W., Nandanwar, D.V., Dhakate, S.R.: Carbon nanotubes reinforced conducting polyaniline and its derivative poly (o-anisidine) composites. Adv. Mater. Lett. 4, 35-38 (2013)

21. Wang, X., Deng, J., Duan, X., Liu, D., Guo, J., Liu, P.: Crosslinked polyaniline nanorods with improved electrochemical performance as electrode material for supercapacitors. J. Mater. Chem. A 2, 12323-12329 (2014)

Publisher's Note Springer Nature remains neutral with regard to jurisdictional claims in published maps and institutional affiliations. 